\title{
P02-56
}

\section{DO STRESSFUL LIFE EVENTS PREDICT EATING DISORDER RELAPSE?: SIX-YEAR OUTCOMES FROM THE COLLABORATIVE PERSONALITY DISORDERS STUDY}

\author{
C. Grilo ${ }^{1}$, M. Pagano ${ }^{2}$, R. Stout ${ }^{3}$
}

${ }^{1}$ Psychiatry, Yale University School of Medicine, New Haven, ${ }^{2}$ Psychiatry, Case Western Reserve University, Cleveland, ${ }^{3}$ Psychiatry, Brown University, Providence, USA

\begin{abstract}
Aims: To examine the natural course of eating disorders (ED) prospectively over 6 years and to examine link between stressful life events (SLEs) and ED relapse among women with personality-disorders (PDs).

Method: Subjects were 132 female patients with bulimia nervosa $(\mathrm{N}=40)$ or EDNOS $(\mathrm{N}=92)$ in the Collaborative Longitudinal Personality Disorders Study. EDs were assessed with the Structured Clinical Interview for DSM-IV Axis-I, and monitored with the Longitudinal Interval Follow-up Evaluation during follow-up. PDs were assessed with the Diagnostic Interview for DSM-IV PD (DIPDIV), and monitored with the Follow-Along version of the DIPD-IV during follow-up. SLEs were assessed with the Life Events Assessment (LEA). Follow-up assessments were conducted at 6- and 12-months and then yearly through 72 months. Proportional hazard regression analyses were performed to examine the link between time-varying levels of SLEs and ED relapse. Cox regressions controlled for the same covariates used in prior work: duration of ED, number of co-morbid psychiatric disorders, and time-varying status of PDs.
\end{abstract}

Results: Of the 132 patients, 59\% had remissions from ED, $68 \%$ of whom subsequently relapsed over the course of 6 years (BN and EDNOS did not differ in relapse). Total number of negative SLEs reported by ED patients significantly predicted subsequent ED relapse (HazardRatio=1.5, $\mathrm{p}<.05)$. The types of SLEs that predicted ED relapse were elevated work stressors $($ HazardRatio=3.0, $p<$ $.01)$ and elevated recreation stressors (HazardRatio=3.1, $p<.05)$.

Conclusion: Higher work stress and higher recreation stress represent significant warning signs for triggering relapse for women in remission from $B N$ and EDNOS. 